\title{
Internal model based tracking and disturbance rejection for stable well-posed systems
}

\author{
Richard REBARBER \\ Department of Mathematics and Statistics \\ University of Nebraska - Lincoln \\ Lincoln, NE 68588-0323, USA \\ e-mail: rrebarbe@math.unl.edu \\ George WEISS \\ Dept. of Electrical and Electronic Engineering \\ Imperial College of Science, Technology and Medicine \\ Exhibition Road, London SW7 2BT, UK \\ e-mail: G.Weiss@ic.ac.uk
}

\begin{abstract}
In this paper we solve the tracking and disturbance rejection problem for infinite-dimensional linear systems, with reference and disturbance signals that are superpositions of sinusoids. In one approach we use a low gain controller suggested by the internal model principle. In this approach, our results are a partial extension of results by T. Hämäläinen and S. Pohjolainen. In their papers, the plant is required to have an exponentially stable transfer function in the Callier-Desoer algebra, while in this paper we only require the transfer function of the plant to be exponentially stable and well-posed. The conditions for a transfer function to be well-posed are sufficiently unrestrictive to be verifiable for many partial differential equations in more than one space variable. Our second approach concerns the case when the second component of the plant transfer function (from control input to tracking error) is positive. In this case, we identify a very simple stabilizing controller which is again an internal model, but which does not require low gain. We apply our results to two problems involving systems modeled by partial differential equations: the problem of rejecting external noise in a model for structure/acoustics interactions, and a similar problem for two coupled beams.
\end{abstract}

Keywords: well-posed linear system; tracking; internal model principle; input-output stability; exponential stability; dynamic stabilization; structural acoustics; positive transfer function; optimizability. 


\section{Introduction and statement of the main results}

In this paper we solve a tracking and disturbance rejection problem for stable well-posed linear systems, using a low gain controller suggested by the internal model principle. These results are a partial extension of those in Hämäläinen and Pohjolainen [15] (detailed comments on this connection will be given).

We assume that the plant $\Sigma_{p}$ is a well-posed linear system (as defined in Salamon [30], Staffans [32] or Weiss [34]) and it is exponentially stable. The plant has two inputs, $w$ and $u$. The input $w$ consists of the external signals (references and disturbances) and $u$ is the control input. These signals take values in the Hilbert spaces $W$ and $U$, respectively. The output signal of $\Sigma_{p}$, denoted by $z$, which represents the tracking error, takes values in the Hilbert space $Y$. The transfer function of the plant is

$$
\mathbf{P}=\left[\begin{array}{ll}
\mathbf{P}_{1} & \mathbf{P}_{2}
\end{array}\right]
$$

where $\mathbf{P}_{1}(s) \in \mathcal{L}(W, Y)$ and $\mathbf{P}_{2}(s) \in \mathcal{L}(U, Y)$. Let $\mathbf{C}$ be the transfer function of the well-posed controller $\Sigma_{c}$ which is to be determined, where $\mathbf{C}(s) \in \mathcal{L}(Y, U)$. The connection between these systems and signals is shown in Figure 1.

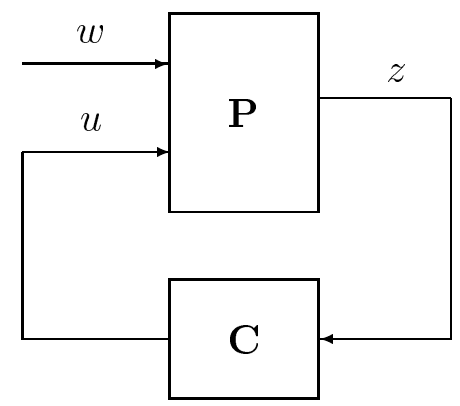

Figure 1: The stable well-posed plant $\mathbf{P}$ with the controller $\mathbf{C}$. The signal $w$ contains disturbances and references, and $z$ is the error signal which should be made small.

Let $\mathcal{J}$ be a finite index set of integers. We assume that $w$ is of the form

$$
w(t)=\sum_{j \in \mathcal{J}} \mathrm{w}_{j} e^{i \omega_{j} t}, \quad \mathrm{w}_{j} \in W, \quad \omega_{j} \in \mathbb{R} .
$$

Thus, $w$ is a superposition of constant and sinusoidal signals. The frequencies $\omega_{j}$ are assumed to be known (for design purposes), but the vectors $\mathrm{w}_{j}$ (which determine the amplitudes and the phases) are not known in advance.

We introduce some terminology and notation. For any $a \in \mathbb{R}$, we put

$$
\mathbb{C}_{a}:=\{s \in \mathbb{C} \mid \operatorname{Re} s>a\}
$$


For any Banach space $Z$, we denote by $H_{a}^{\infty}(Z)$ the space of bounded analytic $Z$ valued functions on $\mathbb{C}_{a}$. For $a=0$ we also use the notation $H^{\infty}(Z)$. In the sequel, when the space $Z$ is clear from the context, we write $H_{a}^{\infty}$ for $H_{a}^{\infty}(Z)$. If a well-posed system is exponentially stable, then its transfer function is in $H_{a}^{\infty}$ for some $a<0$. In particular, for some $a<0$ we have

$$
\mathbf{P}_{1} \in H_{a}^{\infty}(\mathcal{L}(W, Y)), \quad \mathbf{P}_{2} \in H_{a}^{\infty}(\mathcal{L}(U, Y))
$$

A transfer function is called stable if it is in $H^{\infty}$. For any $\alpha \in \mathbb{R}$, set

$$
L_{\alpha}^{2}[0, \infty)=\left\{f \in L_{l o c}^{2}[0, \infty) \mid \int_{0}^{\infty} e^{-2 \alpha t}\|f(t)\|^{2} \mathrm{~d} t<\infty\right\}
$$

The corresponding space of $Y$-valued functions is denoted by $L_{\alpha}^{2}([0, \infty), Y)$ (or also $L^{2}([0, \infty), Y)$ if $\left.\alpha=0\right)$. However, by some abuse of notation, we sometimes just write $L_{\alpha}^{2}[0, \infty)$ when the range space $Y$ is clear from the context.

The objective of this paper is to find a controller $\Sigma_{c}$ with transfer function $\mathbf{C}$ so that the closed-loop system in Figure 1 is exponentially stable, and the output $z$ (the tracking error) decays exponentially to zero, by which we mean that $z \in L_{\alpha}^{2}[0, \infty)$ for some $\alpha<0$. From an engineering point of view, it would look more convincing if we would require that $\lim _{t \rightarrow \infty} z(t)=0$. However, such an objective is unrealistic due to the very general class of plants that we consider. Indeed, the output of $\Sigma_{p}$ resulting from a non-smooth initial state may be in $L_{\alpha}^{2}$ without any further continuity properties, so that point-evaluations of $z$ do not make sense. However, the condition $z \in L_{\alpha}^{2}[0, \infty)$ with $\alpha<0$ actually expresses a very rapid decay of the output, since the sequence $E_{n}=\int_{n}^{n+1}\|z(t)\|^{2} \mathrm{~d} t$ decays exponentially.

We now recall the main result of Hämäläinen and Pohjolainen [15]. Their block diagram is slightly different, it corresponds to taking $\mathbf{P}_{1}=\left[\begin{array}{ll}-I & \mathbf{P}_{2}\end{array}\right]$ in Figure 1, and they consider $U$ and $Y$ to be finite-dimensional, but these are not essential restrictions. They consider $\mathbf{P}$ to be an exponentially stable transfer function in the Callier-Desoer algebra. The internal model principle of Davison, Wonham and Francis (see $[11,12,13]$ ) suggests that $\mathbf{C}$ should have poles at $\left\{i \omega_{j} \mid j \in \mathcal{J}\right\}$. Following this principle, but also relying on a deep insight of their own, the following controller has been proposed by Hämäläinen and Pohjolainen in [15]:

$$
\mathbf{C}(s)=-\varepsilon \sum_{j \in \mathcal{J}} \frac{K_{j}}{s-i \omega_{j}},
$$

with

$$
K_{j} \in \mathcal{L}(Y, U), \quad \sigma\left(\mathbf{P}_{2}\left(i \omega_{j}\right) K_{j}\right) \subset \mathbb{C}_{0} .
$$

Note that (1.3) implies that the range of $\mathbf{P}_{2}\left(i \omega_{j}\right)$ is $Y$ (i.e., the matrix $\mathbf{P}_{2}(i \omega)$ is onto at the relevant frequencies). It was shown in [15] that for all $\varepsilon>0$ sufficiently small, the feedback system in Figure 1 has exponentially stable transfer functions and moreover, if $w$ is as in (1.1), then the error $z$ tends to zero. The approach of [15] is algebraic and they consider also multiple poles in $\mathbf{C}$, which are needed if 
we have polynomial coefficients $\mathrm{w}_{j}$ in (1.1) (we have not reproduced the formulas corresponding to the multiple poles, since we only consider constant $\mathrm{w}_{j}$ ).

This result is important because it allows tracking and disturbance rejection for external signals that are superpositions of a constant and several sinusoidal signals, with very little information about the plant. Indeed, all we have to know is that the plant is stable and we must have some knowledge about $\mathbf{P}_{2}\left(i \omega_{j}\right)$, which can be measured, but we do not need to be very precise (since the conditions (1.3) are robust with respect to small changes of $\left.\mathbf{P}_{2}\left(i \omega_{j}\right)\right)$. By contrast, the internal model theory developed in many references requires a detailed knowledge of the plant equations. We feel that this is an important and beautiful result, and we wanted to understand it from a different perspective, using an analytic approach.

How is our setup different from the one in [15]: the small differences in the block diagram and in the spaces $U, Y$ have already been mentioned (in both respects, our framework is slightly more general). We have constant coefficients $\mathrm{w}_{j}$ in (1.1), so we consider only simple poles for $\mathbf{C}$, and in this respect we are less general. Most importantly, we replace the class of plants in [15] with exponentially stable wellposed plants, which is substantially more general than the class of stable plants with transfer functions in the Callier-Desoer algebra. The conditions for a plant to be well-posed are sufficiently unrestrictive to be verifiable for many partial differential equations in more than one space variable; this is probably not true if we have to verify that the transfer is in the Callier-Desoer algebra.

The transfer function of a well-posed linear system is in $H_{a}^{\infty}$ for any $a \in \mathbb{R}$ which is larger than the growth bound of the operator semigroup of the system, see [34]. Furthermore, any operator-valued function which is in $H_{a}^{\infty}$ for some $a \in \mathbb{R}$ has a realization which is a well-posed linear system, see Salamon [31] and Staffans [33]. Moreover, it follows from the material in [31,33] that if $a<0$, then the realization can be chosen to be exponentially stable. One of our main results follows:

Theorem 1.1. Suppose that $\Sigma_{p}$ is an exponentially stable well-posed linear system with transfer function $\mathbf{P}=\left[\mathbf{P}_{1} \mathbf{P}_{2}\right]$, where $\mathbf{P}_{1}(s) \in \mathcal{L}(W, Y), \mathbf{P}_{2}(s) \in \mathcal{L}(U, Y)$. Let $\Sigma_{c}$ be an exactly controllable and exactly observable realization of a transfer function C of the form

$$
\mathbf{C}(s)=-\varepsilon\left(\mathbf{C}_{0}(s)+\sum_{j \in \mathcal{J}} \frac{K_{j}}{s-i \omega_{j}}\right),
$$

where $\mathbf{C}_{0} \in H_{\alpha}^{\infty}(\mathcal{L}(Y, U))$ with $\alpha<0, K_{j} \in \mathcal{L}(Y, U)$ and $\sigma\left(\mathbf{P}_{2}\left(i \omega_{j}\right) K_{j}\right) \subset \mathbb{C}_{0}$.

Then the feedback system in Figure 1 is exponentially stable for all sufficiently small $\varepsilon>0$. If $w$ is if the form (1.1), then $z \in L_{\beta}^{2}[0, \infty)$ for some $\beta<0$.

Note that, in (1.4), we have added the extra term $\mathbf{C}_{0}$ (when compared to (1.2)). The theorem would of course remain valid without this extra term, but $\mathbf{C}_{0}$ may be needed to satisfy some other design requirements, possibly derived from robustness considerations, or to shorten the transient response. 
This theorem has not been stated in the strongest possible form: it is a consequence of a more general theorem stated and proved in Section 2. In the more general version, the conditions "exactly controllable" and "exactly observable" are replaced with the less restrictive conditions "optimizable" and "estimatable". The reason for not stating this version here is that the concepts of optimizability and estimatability would probably be less familiar to most readers. If $U$ and $Y$ are finite-dimensional and $\mathbf{C}$ is rational (as it would be in most applications), then it is natural to take $\Sigma_{c}$ to be a minimal realization of $\mathbf{C}$, so that $\Sigma_{c}$ would be controllable and observable, as required in the theorem.

Let us consider two additional artificial input signals injected to the feedback system in Figure 1, i.e., signals added to $u$ and $z$. It follows from Theorem 1.1 that (for sufficiently small $\varepsilon$ ) all the resulting transfer functions in Figure 1 (from $w$ and the two new input signals to any other signal) are in $H_{\beta}^{\infty}$ for some $\beta<0$.

We make some comments on the generality of the block diagram in Figure 1 and the signal $w$ in (1.1). Typically $w$ will contain both the reference $r$ and the disturbance $d$, i.e., $w=\left[\begin{array}{ll}r & d\end{array}\right]^{T}$. In this case $r(t) \in Y$ and $d(t)$ is in another Hilbert space $V$, we have $W=Y \times V$ and $\mathbf{P}_{1}$ can be decomposed as $\mathbf{P}_{1}=\left[\begin{array}{ll}-I & \mathbf{P}_{1}^{0}\end{array}\right]$, so

$$
\hat{z}=\mathbf{P}_{1} \hat{w}+\mathbf{P}_{2} \hat{u}=-\hat{r}+\mathbf{P}_{1}^{0} \hat{d}+\mathbf{P}_{2} \hat{u}=\hat{y}-\hat{r},
$$

where $\hat{y}=\mathbf{P}_{1}^{0} \hat{d}+\mathbf{P}_{2} \hat{u}$ is the Laplace transform of the output signal of the plant with transfer function $\mathbf{P}^{0}=\left[\begin{array}{ll}\mathbf{P}_{1}^{0} & \mathbf{P}_{2}\end{array}\right]$, as shown in Figure 2. The paper [15] uses a block diagram which corresponds to this decomposition, with the additional restriction $\mathbf{P}_{1}^{0}=\mathbf{P}_{2}$. In the theory that we develop, such a decomposition of $w$ and of $\mathbf{P}_{1}$ is not needed. We have assumed that the plant is exponentially stable, but in applications $\mathbf{P}$ might be obtained by stabilizing an initially unstable plant.

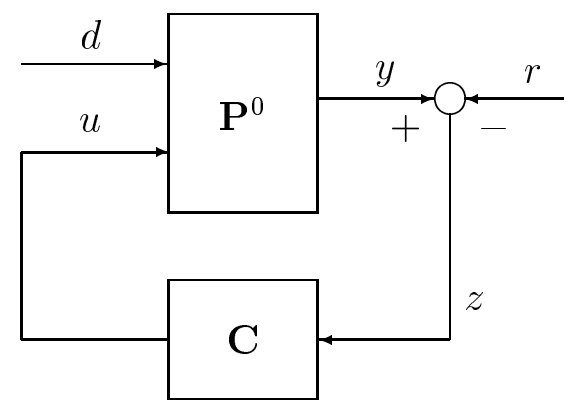

Figure 2: The stable well-posed plant $\mathbf{P}^{0}$ with the controller $\mathbf{C}$. If we lump together the disturbance $d$ and the reference $r$ to form the signal $w$, then this block diagram reduces to the one in Figure 1 , with $\mathbf{P}_{1}=\left[\begin{array}{ll}-I & \mathbf{P}_{1}^{0}\end{array}\right]$.

Usually, $W$ is the complexification of a real Hilbert space $W_{0}$, so that any $\mathrm{w} \in W$ has a unique decomposition $\mathrm{w}=\mathrm{w}^{0}+i \mathrm{w}^{1}$ with $\mathrm{w}^{0}, \mathrm{w}^{1} \in W_{0}$ and so the complex 
conjugate $\overline{\mathrm{w}}=\mathrm{w}^{0}-i \mathrm{w}^{1}$ is well defined. Moreover, the signal $w$ takes values in $W_{0}$, which (with proper indexing) implies that

$$
\omega_{-j}=-\omega_{j}, \quad \text { and } \quad \mathrm{w}_{-j}=\overline{\mathrm{w}}_{j} .
$$

Similarly, $U$ and $Y$ are usually the complexifications of real Hilbert spaces $U_{0}$ and $Y_{0}$ and the transfer function $\mathbf{P}$ is real, which means that

$$
\mathbf{P}_{1}(-i \omega) \mathrm{w}_{0}=\overline{\mathbf{P}_{1}(i \omega) \mathrm{w}_{0}} \quad \forall \mathrm{w}_{0} \in W_{0},
$$

and a similar condition is satisfied by $\mathbf{P}_{2}$. In this case, the controller transfer function $\mathbf{C}$ can be chosen to be real as well, by choosing

$$
K_{-j} \mathrm{y}_{0}=\overline{K_{j} \mathrm{y}_{0}} \quad \forall \mathrm{y}_{0} \in Y_{0}
$$

and by choosing $\mathbf{C}_{0}$ to be real. These restrictions on $w, \mathbf{P}$ and $\mathbf{C}$ had to be mentioned because they would probably arise in any engineering application. However, these restrictions are not necessary for our theory, so that we will not make them. Taking them into account would not modify the theory at all.

The results of Hämäläinen and Pohjolainen [15] build on earlier work by the same authors, notably [26] and [14]. Low-gain tracking results for infinite-dimensional systems can be found in Logemann, Bontsema, and Owens [19], Logemann and Curtain [20], Logemann and Mawby [21], Logemann and Owens [22], Logemann and Townley [24, 25], Logemann, Ryan and Townley [23], Pohjolainen [26, 27], and Pohjolainen and Lätti [28]. In [20, 21, 23, 24, 25] the reference and disturbance signals are constant and the class of plants considered is the class of regular systems, which is a very general subset of well-posed systems. In [20, 21, 23] the systems are allowed to have nonlinearities. In [24] the plants are uncertain and it is shown how the gain can be chosen adaptively. In [25] analogous results are obtained for uncertain discrete-time systems, with the gain obtained adaptively; these results are applied to continuous-time regular systems to obtain adaptive sampled-data low-gain tracking controllers. The references [19, 22, 26, 27, 28] are earlier works where the classes of systems considered are substantially more restrictive than here, and the references and disturbances are constant.

Let $\mathbf{P}$ be an $\mathcal{L}(U)$-valued transfer function defined on (a set containing) the halfplane $\mathbb{C}_{0}$. We say that $\mathbf{P}$ is a positive transfer function if

$$
\operatorname{Re} \mathbf{P}(s):=\frac{1}{2}\left[\mathbf{P}(s)+\mathbf{P}(s)^{*}\right] \geq 0 \quad \forall s \in \mathbb{C}_{0} .
$$

When the second component of the plant transfer function (from control input to error) is positive, the following theorem states that certain simple controllers will stabilize the system in the sense of Theorem 1.1 and also achieve tracking. Moreover, in this situation, there is no need to adjust an unknown small gain.

Theorem 1.2. Suppose that $\Sigma_{p}$ is an exponentially stable well-posed linear system

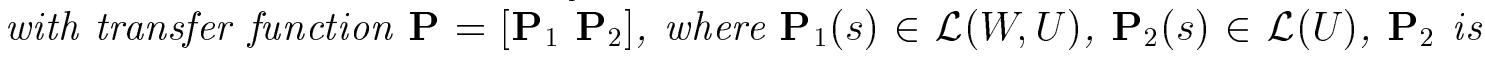


a positive transfer function, and $\operatorname{Re} \mathbf{P}_{2}\left(i \omega_{j}\right)$ is invertible for all $j \in \mathcal{J}$. Let $\Sigma_{c}$ be an exactly controllable and exactly observable realization of a transfer function $\mathbf{C}$ of the form

$$
\mathbf{C}(s)=-\left(\mathbf{C}_{0}(s)+\sum_{j \in \mathcal{J}} \frac{K_{j}}{s-i \omega_{j}}\right),
$$

where $K_{j} \in \mathcal{L}(U), K_{j}>0, K_{j}^{-1} \in \mathcal{L}(U), \mathbf{C}_{0} \in H_{\alpha}^{\infty}(\mathcal{L}(U))$ with $\alpha<0$ and

$$
\operatorname{Re} \mathbf{C}_{0}(s) \geq \frac{1}{2} I \quad \forall s \in \mathbb{C}_{0}
$$

Then the feedback system in Figure 1 is exponentially stable. If $w$ is if the form (1.1), then $z \in L_{\beta}^{2}[0, \infty)$ for some $\beta<0$.

Concerning the conditions "exactly controllable" and "exactly observable" that appear in this theorem, the same comments apply as those made after Theorem 1.1. The more general version of Theorem 1.2 is stated and proved in Section 2.

We remark that a simple choice for $K_{j}$ and $\mathbf{C}_{0}$ is to take them scalar multiples of the identity operator. Then $\mathbf{C}$ is essentially a single input-single output system repeated in identical copies as many times as the dimension of $U$. This choice still allows $\mathbf{C}_{0}$ to be a function of $s$, but of course the simplest choice for $\mathbf{C}_{0}$ would be to take it a real constant larger than 0.5 .

In Section 3 we apply Theorem 1.1 to a model for structure/acoustics interaction, showing that external noise at fixed frequencies can be rejected. In Section 4 we apply Theorem 1.2 to a model for coupled Euler-Bernoulli beams.

\section{Proof of the main results}

To prove the stability parts of Theorems 1.1 and 1.2 we show that an appropriate set of closed-loop transfer functions is stable, and then we apply results from Weiss and Rebarber [37] to conclude that the closed-loop system is exponentially stable. We will need the concepts of optimizability and estimatability, as defined in Sections 3 and 4 of [37]. Without going into the details of notation, a well-posed system is called optimizable if for any initial state, an input function in $L^{2}[0, \infty)$ can be found such that the state trajectory is in $L^{2}[0, \infty)$. Motivated by linear quadratic optimal control theory, this property is sometimes called the finite cost condition. This is one possible infinite-dimensional generalization of the well-known concept of stabilizability from finite-dimensional control theory. Estimatability is the dual concept: it can be formulated directly, but it will suffice here to say that a wellposed system is called estimatable if its dual system is optimizable. Thus, this is one possible infinite-dimensional generalization of detectability. An exponentially stable system is automatically optimizable and estimatable. We note that any exactly controllable system is optimizable, and any exactly observable system is estimatable. The converse of these statements is not true. 
Instead of directly quoting results from [37] in our proofs of the main theorems, it will be easier to follow our arguments if we formulate and use the following lemma, which is a direct consequence of a result in [37] (related results for regular linear systems were given in Weiss and Curtain [36]).

Lemma 2.1. Suppose that $\Sigma_{p}$ and $\Sigma_{c}$ are well-posed linear systems with transfer functions denoted $\mathbf{P}$ and $\mathbf{C}$ (where $\mathbf{P}=\left[\begin{array}{ll}\mathbf{P}_{1} & \mathbf{P}_{2}\end{array}\right]$ ), connected in feedback as shown in Figure 1. Suppose that $\Sigma_{p}$ is optimizable via $u, \Sigma_{c}$ is optimizable, and both systems are estimatable. Suppose that the four transfer functions

$$
\left(I-\mathbf{P}_{2} \mathbf{C}\right)^{-1}, \mathbf{C}\left(I-\mathbf{P}_{2} \mathbf{C}\right)^{-1},\left(I-\mathbf{P}_{2} \mathbf{C}\right)^{-1} \mathbf{P}_{2}, \mathbf{C}\left(I-\mathbf{P}_{2} \mathbf{C}\right)^{-1} \mathbf{P}_{2}
$$

are all stable (i.e., in $H^{\infty}$ ). Then the closed-loop system $\Sigma_{p, c}$ shown in Figure 1 is an exponentially stable well-posed linear system.

Proof. Under the given optimizability and estimatability hypotheses, it follows from [37, Theorem 6.4] that the closed-loop system $\Sigma_{p, c}$ is well-posed and exponentially stable if (and only if) $(I-\mathbf{L})^{-1} \in H^{\infty}$, where

$$
\mathbf{L}=\left[\begin{array}{cc}
0 & \mathbf{C} \\
\mathbf{P}_{2} & 0
\end{array}\right]
$$

It is easy to verify that $(I-\mathbf{L})^{-1} \in H^{\infty}$ if (and only if) the transfer functions listed in (2.1) are all in $H^{\infty}$.

Note that the four transfer functions listed in (2.1) are a subset of the transfer functions that we get if we consider two artificial new inputs for the system in Figure 1 , as mentioned after Theorem 1.1, and we put $w=0$. It is easy to see that these four are stable if and only if all of them (those corresponding to $w=0$ ) are stable.

Remark 2.2. With the assumptions of Lemma 2.1, let $\gamma$ denote the growth bound of the semigroup of $\Sigma_{p, c}$, so that $\gamma<0$ according to the lemma. Then according to [34, Proposition 4.1], all of the transfer functions associated with this system (such as the four transfer functions listed in (2.1)) are in $H_{\beta}^{\infty}$ for any $\beta>\gamma$. Similarly, the transfer function from $w$ to $z$, which is

$$
\mathbf{G}=\left(I-\mathbf{P}_{2} \mathbf{C}\right)^{-1} \mathbf{P}_{1}
$$

is in $H_{\beta}^{\infty}$ for any $\beta>\gamma$.

To be able to apply Lemma 2.1, a key step is to show that the first of the four transfer functions listed in (2.1), called the sensitivity and denoted by $\mathbf{S}$, is stable (i.e., in $H^{\infty}$ ) for all sufficiently small $\varepsilon>0$. 
Lemma 2.3. Suppose that $\mathbf{P}_{2} \in H_{a}^{\infty}(\mathcal{L}(U, Y))$ with $a<0$ and $\mathbf{C}$ is given by (1.4) and satisfies the conditions listed after (1.4). Then there exists $\varepsilon^{*}>0$ such that for all $\varepsilon \in\left(0, \varepsilon^{*}\right]$,

$$
\mathbf{S}:=\left(I-\mathbf{P}_{2} \mathbf{C}\right)^{-1} \in H^{\infty}
$$

Proof: Let $r_{0}>0$ be such that $\left|\omega_{j}-\omega_{k}\right|>2 r_{0}$ for all $j, k \in \mathcal{J}$ with $j \neq k$. We define the half-disks

$$
D_{j}:=\left\{s \in \mathbb{C}|\operatorname{Re} s \geq 0,| s-i \omega_{j} \mid \leq r_{0}\right\}
$$

Since $\mathbf{P}_{2}$ and $\mathbf{C}_{0}$ are bounded on $\mathbb{C}_{0}$ and $\mathcal{J}$ is finite set, it is clear that

$$
\sup _{s \in \mathcal{G}}\left\|\mathbf{P}_{2}(s)\left[\mathbf{C}_{0}(s)+\sum_{j \in \mathcal{J}} \frac{K_{j}}{s-i \omega_{j}}\right]\right\|<\infty,
$$

where

$$
\mathcal{G}=\mathbb{C}_{0} \backslash \cup_{j \in \mathcal{J}} D_{j}
$$

Therefore, there exists $\varepsilon_{\infty}>0$ such that

$$
\mathbf{S}(s)=\left(I+\varepsilon \mathbf{P}_{2}(s)\left[\mathbf{C}_{0}(s)+\sum_{j \in \mathcal{J}} \frac{K_{j}}{s-i \omega_{j}}\right]\right)^{-1}
$$

is uniformly bounded for all $s \in \mathcal{G}$ and all $\varepsilon \in\left(0, \varepsilon_{\infty}\right]$.

Fix $j \in \mathcal{J}$. To analyze $\mathbf{S}$ on $D_{j}$, we define

$$
\mathbf{S}_{j}(s):=\left(I+\frac{\varepsilon \mathbf{P}_{2}\left(i \omega_{j}\right) K_{j}}{s-i \omega_{j}}\right)^{-1} .
$$

(this is a good approximation of $\mathbf{S}(s)$ near the point $s=i \omega_{j}$ ) and

$$
\mathbf{Q}_{j}(s)=\frac{\left(\mathbf{P}_{2}(s)-\mathbf{P}_{2}\left(i \omega_{j}\right)\right) K_{j}}{s-i \omega_{j}}+\mathbf{P}_{2}(s) \mathbf{C}_{0}(s)+\sum_{k \in \mathcal{J}, k \neq j} \frac{\mathbf{P}_{2}(s) K_{k}}{s-i \omega_{k}}
$$

Then $\mathbf{S}^{-1}-\mathbf{S}_{j}^{-1}=\varepsilon \mathbf{Q}_{j}$, so that we can write

$$
\mathbf{S}(s)=\mathbf{S}_{j}(s)\left(I+\varepsilon \mathbf{Q}_{j}(s) \mathbf{S}_{j}(s)\right)^{-1} .
$$

It is clear that the last two terms in the definition of $\mathbf{Q}_{j}$ are bounded on $D_{j}$. Since $\mathbf{P}_{2}$ is analytic at $i \omega_{j}$, the first term in the definition of $\mathbf{Q}_{j}$ is also bounded on $D_{j}$. Therefore $\mathbf{Q}_{j}$ is bounded on $D_{j}$, with a bound that is independent of $\varepsilon$ (because $\mathbf{Q}_{j}$ is independent of $\varepsilon$ ). If we can show that $\mathbf{S}_{j}$ is bounded on $D_{j}$, with the bound independent of $\varepsilon>0$, then from (2.2) we see that there exists $\varepsilon_{j}>0$ such that $\mathbf{S}$ 
is bounded on $D_{j}$ for all $\varepsilon \in\left(0, \varepsilon_{j}\right]$. Since $\mathcal{J}$ has finitely many elements, this will finish the proof, with

$$
\varepsilon^{*}=\min \left\{\varepsilon_{j} \mid j \in \mathcal{J}\right\} \cup\left\{\varepsilon_{\infty}\right\}
$$

To show that $\mathbf{S}_{j}$ is bounded on $D_{j}$, with the bound independent of $\varepsilon>0$, denote $P_{j}=\mathbf{P}_{2}\left(i \omega_{j}\right) K_{j}, z=\left(s-i \omega_{j}\right) / \varepsilon$, and note that

$$
\begin{gathered}
\sup _{s \in D_{j}}\left\|\mathbf{S}_{j}(s)\right\|=\sup _{\operatorname{Re} z \geq 0,|z| \leq r / \varepsilon}\left\|\left(I+\frac{P_{j}}{z}\right)^{-1}\right\| \\
\leq \sup _{\operatorname{Re} z \geq 0}\left\|z\left(z I+P_{j}\right)^{-1}\right\| .
\end{gathered}
$$

Since $\sigma\left(P_{j}\right) \subset \mathbb{C}_{0}, z\left(z I+P_{j}\right)^{-1}$ is analytic on $\overline{\mathbb{C}_{0}}$. Moreover,

$$
\lim _{|z| \rightarrow \infty} z\left(z I+P_{j}\right)^{-1}=I
$$

so $z\left(z I+P_{j}\right)^{-1}$ is continuous on the one-point compactification of $\overline{\mathbb{C}_{0}}$, hence it is bounded there. Therefore $\mathbf{S}_{j}$ is bounded on $D_{j}$ with a bound independent of $\varepsilon$, which (as explained earlier) finishes the proof of the lemma.

Theorem 1.1 is an immediate consequence of the following, more general theorem.

Theorem 2.4. Let $\Sigma_{p}$ be an exponentially stable well-posed linear system with transfer function $\mathbf{P}=\left[\begin{array}{ll}\mathbf{P}_{1} & \mathbf{P}_{2}\end{array}\right]$, where $\mathbf{P}_{1}(s) \in \mathcal{L}(W, Y), \mathbf{P}_{2}(s) \in \mathcal{L}(U, Y)$. Let $\Sigma_{c}$ be an optimizable and estimatable realization of a transfer function $\mathbf{C}$ of the form (1.4), where $\mathbf{C}_{0} \in H_{\alpha}^{\infty}(\mathcal{L}(Y, U))$ with $\alpha<0, K_{j} \in \mathcal{L}(Y, U)$ and $\sigma\left(\mathbf{P}_{2}\left(i \omega_{j}\right) K_{j}\right) \subset \mathbb{C}_{0}$.

Then the feedback system in Figure 1 is exponentially stable for all sufficiently small $\varepsilon>0$. If $w$ is if the form (1.1), then $z \in L_{\beta}^{2}[0, \infty)$ for some $\beta<0$.

The simplest way to obtain an optimizable and estimatable realization of $\mathbf{C}$ is to take an exponentially stable realization of $-\varepsilon \mathbf{C}_{0}$ and connect it in parallel with a minimal (i.e., exactly observable and exactly controllable) realization of the rational transfer function $\mathbf{C}+\varepsilon \mathbf{C}_{0}$. Note that this minimal realization is not finitedimensional in general, because the coefficients $K_{j}$ are operators.

Proof. Since $\Sigma_{p}$ is exponentially stable, we have $\mathbf{P} \in H_{a}^{\infty}$ with $a<0$. According to Lemma 2.3, for $\varepsilon \in\left(0, \varepsilon^{*}\right]$ we have $\left(I-\mathbf{P}_{2} \mathbf{C}\right)^{-1} \in H^{\infty}$. We need to show that for such $\varepsilon$, the other transfer functions listed in (2.1) are also in $H^{\infty}$. Due to the stability of $\mathbf{P}_{2}$, this follows once we have shown that

$$
\mathbf{C}\left(I-\mathbf{P}_{2} \mathbf{C}\right)^{-1} \in H^{\infty}
$$

To prove this, we notice that by (1.4), $\lim _{s \rightarrow i \omega_{j}}\left(s-i \omega_{j}\right) \mathbf{C}(s)=-\varepsilon K_{j}$ for any $j \in \mathcal{J}$. This implies that

$$
\lim _{s \rightarrow i \omega_{j}} \frac{1}{s-i \omega_{j}}\left[I-\mathbf{P}_{2}(s) \mathbf{C}(s)\right]^{-1}
$$




$$
=\left[\lim _{s \rightarrow i \omega_{j}} \mathbf{P}_{2}(s)\left(s-i \omega_{j}\right) \mathbf{C}(s)\right]^{-1}=-\frac{1}{\varepsilon}\left[\mathbf{P}_{2}\left(i \omega_{j}\right) K_{j}\right]^{-1} .
$$

Note that $\mathbf{P}_{2}\left(i \omega_{j}\right) K_{j}$ is invertible according to (1.3). Now, using again the decomposition (1.4), we conclude that $\mathbf{C}\left(I-\mathbf{P}_{2} \mathbf{C}\right)^{-1}$ has a finite limit at $i \omega_{j}$, for all $j \in \mathcal{J}$, so that $\mathbf{C}\left(I-\mathbf{P}_{2} \mathbf{C}\right)^{-1}$ is bounded on a neighborhood $\mathcal{N}$ of the set $\left\{i \omega_{j} \mid j \in \mathcal{J}\right\}$. Since $\left(I-\mathbf{P}_{2} \mathbf{C}\right)^{-1} \in H^{\infty}$ and $\mathbf{C}$ is uniformly bounded on $\mathbb{C}_{0} \backslash \mathcal{N}$, it follows that $\mathbf{C}\left(I-\mathbf{P}_{2} \mathbf{C}\right)^{-1} \in H^{\infty}$. As mentioned earlier, this implies that all of the transfer functions listed in (2.1) are in $H^{\infty}$.

Because $\Sigma_{p}$ is exponentially stable, it is optimizable and estimatable. $\Sigma_{c}$ is optimizable and estimatable by hypothesis. Now we apply Lemma 2.1 to conclude that $\Sigma_{p, c}$ is exponentially stable, so that its semigroup has growth bound $\gamma<0$.

Now we choose $\beta \in(\gamma, 0)$ and we prove that $z \in L_{\beta}^{2}[0, \infty)$. First we recall from Remark 2.2 that the transfer function $\mathbf{G}$ from $w$ to $z$ is in $H_{\beta}^{\infty}$. We compute, using $(2.3)$

$$
\lim _{s \rightarrow i \omega_{j}} \frac{1}{s-i \omega_{j}} \mathbf{G}(s)=-\frac{1}{\varepsilon}\left[\mathbf{P}_{2}\left(i \omega_{j}\right) K_{j}\right]^{-1} \mathbf{P}_{1}\left(i \omega_{j}\right) .
$$

According to (1.1), we have

$$
\hat{w}(s)=\sum_{j \in \mathcal{J}} \frac{\mathrm{w}_{j}}{s-i \omega_{j}} .
$$

This, together with (2.4), shows that $\mathbf{G} \hat{w}$ has removable singularities at the points $i \omega_{j}, j \in \mathcal{J}$. Since $\mathbf{G}$ is bounded on $\mathbb{C}_{\beta}$ and $\hat{w}$ decays like $1 / s$ for large $|s|$, we see that $\mathbf{G} \hat{w} \in H_{\beta}^{2}$. Here $H_{\beta}^{2}$ denotes the space of those ( $Y$-valued) analytic functions on $\mathbb{C}_{\beta}$ which can be obtained by shifting a function in the Hardy space $H^{2}$ (on $\mathbb{C}_{0}$ ) to the left by $|\beta|$. Equivalently, by the Paley-Wiener theorem, $H_{\beta}^{2}$ is the space of Laplace transforms of functions in $L_{\beta}^{2}[0, \infty)$.

The output function $z$ of $\Sigma_{p, c}$ can be decomposed as $z=z_{1}+z_{2}$, where $z_{1}$ is the component due to the initial state and $z_{2}$ is the component due to the input $w$. According to $\left[34\right.$, formula (4.2)], $z_{1} \in L_{\beta}^{2}[0, \infty)$. Since $\hat{z}_{2}=\mathbf{G} \hat{w} \in H_{\beta}^{2}$, we have that $z_{2} \in L_{\beta}^{2}[0, \infty)$. Thus we have shown that $z \in L_{\beta}^{2}[0, \infty)$.

We now turn our attention to the proof of Theorem 1.2. We will need the following simple lemma, which is related to the computations usually found in the theory of the Cayley transform, but we are not aware of a reference for this lemma.

Lemma 2.5. Suppose that $H$ is a Hilbert space and $\mathbf{T} \in \mathcal{L}(H)$. Then

$$
\operatorname{Re} \mathbf{T} \geq \frac{1}{2} I
$$

if and only if there exists $\mathbf{Q} \in \mathcal{L}(H)$ such that

$$
\mathbf{T}=(I-\mathbf{Q})^{-1}, \quad \text { with } \quad\|\mathbf{Q}\| \leq 1 .
$$

Furthermore, if the above conditions are satisfied and we denote $M=\|\mathbf{T}\|$, then

$$
\operatorname{Re} \mathbf{Q} \leq\left(1-\frac{1}{2 M^{2}}\right) I .
$$


Proof. Suppose that $\operatorname{Re} \mathbf{T} \geq \frac{1}{2} I$. Then for any $\mathrm{u} \in H$ with $\|\mathrm{u}\|=1$,

$$
\|\mathbf{T u}\| \geq|\langle\mathbf{T u}, \mathrm{u}\rangle| \geq \operatorname{Re}\langle\mathbf{T u}, \mathrm{u}\rangle=\langle(\operatorname{Re} \mathbf{T}) \mathrm{u}, \mathrm{u}\rangle \geq \frac{1}{2},
$$

so that $\mathbf{T}$ is bounded from below. By a similar argument, $\mathbf{T}^{*}$ is also bounded from below, hence $\mathbf{T}$ is onto, so that $\mathbf{T}$ is invertible. We have for any $\mathrm{v} \in H$

$$
\begin{aligned}
\|(\mathbf{T}-I) \mathrm{v}\|^{2} & =\langle(\mathbf{T}-I) \mathrm{v},(\mathbf{T}-I) \mathrm{v}\rangle \\
& =\|\mathbf{T} \mathrm{v}\|^{2}-2 \operatorname{Re}\langle\mathbf{T} \mathrm{v}, \mathrm{v}\rangle+\|\mathrm{v}\|^{2} \\
& \leq\|\mathbf{T} \mathrm{v}\|^{2}-\|\mathrm{v}\|^{2}+\|\mathrm{v}\|^{2}=\|\mathbf{T} \mathrm{v}\|^{2}
\end{aligned}
$$

Denoting $z=\mathbf{T v}_{\mathrm{v}}$, we have shown that

$$
\left\|(\mathbf{T}-I) \mathbf{T}^{-1} z\right\| \leq\|z\| \quad \forall z \in H,
$$

so that $\mathbf{Q}=(\mathbf{T}-I) \mathbf{T}^{-1}=I-\mathbf{T}^{-1}$ is a contraction. It is clear that $\mathbf{T}=(I-\mathbf{Q})^{-1}$

Conversely, suppose that (2.5) holds. Then obviously $\mathbf{T}$ is invertible and $\mathbf{Q}=$ $(\mathbf{T}-I) \mathbf{T}^{-1}$, so that (2.6) holds. Denoting $\mathrm{v}=\mathbf{T}^{-1} z$, we ge

$$
\|(\mathbf{T}-I) \mathrm{v}\| \leq\|\mathbf{T} \mathrm{v}\| \quad \forall \mathrm{v} \in H .
$$

Developing $\|(\mathbf{T}-I) \mathrm{v}\|^{2}$ as in the first part of the proof, we obtain that $2 \operatorname{Re}\langle\mathbf{T v}, \mathrm{v}\rangle \geq$ $\|\mathrm{v}\|^{2}$, which is the same as $\operatorname{Re} \mathbf{T} \geq \frac{1}{2} I$.

The final part of the lemma follows from

$$
\mathbf{Q}^{*}+\mathbf{Q}=2 I-\mathbf{T}^{-*}-\mathbf{T}^{-1}=2 I-\mathbf{T}^{-*}\left(\mathbf{T}^{*}+\mathbf{T}\right) \mathbf{T}^{-1} \leq 2 I-\mathbf{T}^{-*} \mathbf{T}^{-1} .
$$

If $\|\mathbf{T}\|=M$ then $\mathbf{T}^{-*} \mathbf{T}^{-1} \geq \frac{I}{M^{2}}$, so that

$$
\mathrm{Q}^{*}+\mathrm{Q} \leq 2 I-\frac{I}{M^{2}} .
$$

Theorem 1.2 is an immediate consequence of the following, more general theorem.

Theorem 2.6. Suppose that $\Sigma_{p}$ is an exponentially stable well-posed linear system with transfer function $\mathbf{P}=\left[\mathbf{P}_{1} \mathbf{P}_{2}\right]$, where $\mathbf{P}_{1}(s) \in \mathcal{L}(W, U), \mathbf{P}_{2}(s) \in \mathcal{L}(U), \mathbf{P}_{2}$ is a positive transfer function, and $\operatorname{Re} \mathbf{P}_{2}\left(i \omega_{j}\right)$ is invertible for all $j \in \mathcal{J}$. Let $\Sigma_{c}$ be an optimizable and estimatable realization of a transfer function $\mathbf{C}$ of the form (1.5), where $K_{j} \in \mathcal{L}(U), K_{j}>0, K_{j}^{-1} \in \mathcal{L}(U), \mathbf{C}_{0} \in H_{\alpha}^{\infty}(\mathcal{L}(U))$ with $\alpha<0$ and

$$
\operatorname{Re} \mathbf{C}_{0}(s) \geq \frac{1}{2} I \quad \forall s \in \mathbb{C}_{0}
$$

Then the feedback system in Figure 1 is exponentially stable. If $w$ is if the form (1.1), then $z \in L_{\beta}^{2}[0, \infty)$ for some $\beta<0$. 
Proof. For every $K_{j} \in \mathcal{L}(U)$ with $K_{j} \geq 0$ and every $\omega_{j} \in \mathbb{R}$,

$$
\operatorname{Re}\left(\frac{K_{j}}{s-i \omega_{j}}\right) \geq 0 \quad \forall s \in \mathbb{C}_{0} .
$$

Therefore, from the formula (1.5) we see that

$$
-\operatorname{Re} \mathbf{C}(s) \geq \frac{1}{2} I
$$

which implies that for all $s \in \mathbb{C}_{0}$

$$
\|\mathbf{C}(s) \mathrm{v}\| \geq \frac{1}{2}\|\mathrm{v}\| \quad \forall \mathrm{v} \in U .
$$

By Lemma 2.5, (2.7) implies that there exists $\mathbf{Q} \in \mathcal{L}(H)$ such that

$$
-\mathbf{C}=(I-\mathbf{Q})^{-1}, \quad\|\mathbf{Q}\| \leq 1 .
$$

Since $\mathbf{S}=\left(I-\mathbf{P}_{2} \mathbf{C}\right)^{-1}$, we obtain that

$$
\mathbf{S}^{-1}=I+\mathbf{P}_{2}(I-\mathbf{Q})^{-1}=\left[I-\mathbf{Q}+\mathbf{P}_{2}\right](I-\mathbf{Q})^{-1}=-\left[I-\mathbf{Q}+\mathbf{P}_{2}\right] \mathbf{C}
$$

Since by our assumptions $\operatorname{Re} \mathbf{P}_{2}\left(i \omega_{j}\right)$ is positive and invertible for each $j \in \mathcal{J}$, there exists $\varepsilon>0$ and $\delta_{j}>0$ such that for all $j \in \mathcal{J}$,

$$
\operatorname{Re} \mathbf{P}_{2}(s)>\varepsilon I \text { for every } s \in D_{j}:=\left\{s \in \mathbb{C}_{0}|| s-i \omega_{j} \mid<\delta_{j}\right\} .
$$

Since $\operatorname{Re} \mathbf{Q} \leq I$ and $\operatorname{Re} \mathbf{P}_{2} \geq \varepsilon I$ for $s \in D_{j}$, we see that $\operatorname{Re}\left[I-\mathbf{Q}(s)+\mathbf{P}_{2}(s)\right] \geq \varepsilon I$, so that

$$
\left\|\left[I-\mathbf{Q}(s)+\mathbf{P}_{2}(s)\right] \mathrm{u}\right\| \geq \varepsilon\|\mathrm{u}\| \quad \forall \mathrm{u} \in U .
$$

Using this, together with (2.8) and (2.9), we obtain

$$
\left\|\mathrm{S}^{-1}(s) \mathrm{v}\right\| \geq \frac{\varepsilon}{2}\|\mathrm{v}\| \quad \forall \mathrm{v} \in U, s \in \cup_{j \in \mathcal{J}} D_{j} .
$$

Furthermore, $\mathbf{C}$ is bounded on $\mathbb{C}_{0} \backslash \cup_{j \in \mathcal{J}} D_{j}$, so from Lemma 2.5 there exists $\varepsilon_{1}>0$ such that $\operatorname{Re} \mathbf{Q} \leq\left(1-\varepsilon_{1}\right) I$ in $\mathbb{C}_{0} \backslash \cup_{j \in \mathcal{J}} D_{j}$. Since by hypothesis $\operatorname{Re} \mathbf{P}_{2}(s) \geq 0$ for $s \in \mathbb{C}_{0}, \operatorname{Re}\left(I-\mathbf{Q}(s)+\mathbf{P}_{2}(s)\right) \geq \varepsilon_{1} I$ for $s \in \mathbb{C}_{0} \backslash \cup_{j \in \mathcal{J}} D_{j}$. Therefore, using again the formulas (2.8) and (2.9), we obtain that

$$
\left\|\mathbf{S}^{-1}(s) \mathrm{v}\right\| \geq \frac{\varepsilon_{1}}{2}\|\mathrm{v}\| \quad \forall \mathrm{v} \in U, s \in \mathbb{C}_{0} \backslash \cup_{j \in \mathcal{J}} D_{j}
$$

Combining (2.10) and (2.11), we see that $\mathbf{S}^{-1}$ is uniformly bounded from below on $\mathbb{C}_{0}$, i.e., $\mathbf{S}(s)$ is uniformly bounded on $\mathbb{C}_{0}$. Now we can follow the rest of the proof of Theorem 2.4, taking $\varepsilon=1$. 


\section{Noise reduction in a stuctural acoustics model}

The model we consider is based on one in Banks et. al. [7, 8], but we include additional damping, in the form of absorbing boundary conditions on cavity walls. This model is motivated by the following situation: A rectangle $\Omega$ represents a two-dimensional cross section of an acoustic cavity. An acoustic velocity potential $z$ satisfies the wave equation in $\Omega$. $\Gamma_{0}$ is one side of the boundary of $\Omega$, and represents a cross section of the active wall of the cavity. We denote the displacement of this wall by $v$, which satisfies a beam equation with Kelvin-Voigt damping. The boundary conditions for the wave equation include boundary damping which causes the system energy to decay exponentially. The control action is realized by the placement of a piezoelectric ceramic patch on $\Gamma_{0}$; a voltage is applied on this patch and the resulting bending moments can be interpreted as derivatives of delta functions. Furthermore, there is a noise source $d(t)$ applied to the active wall, which we assume to be the sum of finitely many sinusoids; in an application the acoustic cavity might be an airplane cockpit and the noise might be engine noise. We refer to $z_{t}$ as the acoustic pressure, although it is actually proportional to the acoustic pressure. We are interested in reducing the acoustic pressure near a point $x_{0} \in \Omega$, so we take the observation $y(t)$ to be an integral of the acoustic pressure over a small neighborhood $\Omega_{0} \subset \Omega$ of $x_{0}$. We wish to design a controller to maintain the exponential stability of the system, and reject the effect of the noise $d(t)$ from the output. We will apply Theorem 1.1 to obtain this controller.

Let $z=z(t, x)$ for $t \in[0, \infty)$ and $x \in \Omega$, let $v=v(t, \xi)$ for $t \in[0, \infty)$ and $\xi \in \Gamma_{0}$, and let $\partial / \partial \nu$ denote the outward normal derivative on $\Gamma$. Let $U=Y=\mathbb{R}$. In our example $B$ will represent the action of one piezoelectric ceramic patch, and hence be of the form $\alpha_{1} \delta_{\eta_{1}}^{\prime}+\alpha_{2} \delta_{\eta_{2}}^{\prime}$, where $\alpha_{1}, \alpha_{2} \in \mathbb{R}$ and $\eta_{1}, \eta_{2} \in \Gamma_{0}$. In fact, the development here works for any $B \in \mathcal{L}\left(U, H^{-7 / 4}\left(\Gamma_{0}\right)\right)$. Let the disturbance be of the form

$$
d(t)=\sum_{j \in \mathcal{J}} \mathrm{d}_{j} e^{i \omega_{j} t}, \quad \mathrm{~d}_{j} \in \mathbb{R}, \quad \omega_{j} \in \mathbb{R} .
$$

Let $B_{1}(\xi)$ be a function on $\Gamma_{0}$ which describes the spatial distribution on which the disturbance term $d(t)$ acts; for instance, if the noise is uniformly distributed throughout $\Gamma_{0}$, then $B_{1}(\xi)=1$. We will assume for simplicity that $B_{1} \in L^{2}\left(\Gamma_{0}\right)$, although the same procedure will work for some distributions $B_{1}$.

We refer to the following as the structural acoustics model:

$$
\begin{aligned}
z_{t t} & =\Delta z \text { on }[0, \infty) \times \Omega, \\
\frac{\partial z}{\partial \nu}+\beta z_{t} & =v_{t} \quad \text { on }[0, \infty) \times \Gamma_{0} \\
\frac{\partial z}{\partial \nu}+\beta z_{t} & =0 \quad \text { on }[0, \infty) \times \Gamma \backslash \Gamma_{0}, \\
v_{t t} & =-\Delta^{2} v-\Delta^{2} v_{t}-z_{t}+B u+B_{1} d \text { on }[0, \infty) \times \Gamma_{0}, \\
v(t, a) & =v(t, b)=0, \quad \frac{\partial v(t, a)}{\partial x}=\frac{\partial v(t, b)}{\partial x}=0 \quad \text { for } t \in[0, \infty),
\end{aligned}
$$




$$
y(t)=\int_{\Omega_{0}} z_{t}(x, t) d x
$$

The damping, parameterized by $\beta>0$, is either a natural part of the system, such as an energy-absorbing coating, or it would be introduced as a stabilizing feedback before the low-gain tracking is applied. This system fits into the framework described by Figure 2 (which is a special case of the system in Figure 1), where the reference signal $r$ is taken to be zero, $\mathbf{P}_{2}$ is the transfer function from $u$ into $y$, and $\mathbf{P}_{1}^{0}$ is the transfer function from $d$ to $y$. We need to verify the hypotheses of Theorem 1.1.

The state of this system is $x(t)=\left[z, z_{t}, v, v_{t}\right]^{T}$ and the standard state space is

$$
X=\frac{H^{1}(\Omega)}{\mathbb{R}} \times \frac{L^{2}(\Omega)}{\mathbb{R}} \times H_{0}^{2}\left(\Gamma_{0}\right) \times L^{2}\left(\Gamma_{0}\right),
$$

where $L^{2}(\Omega) / \mathbb{R}=\left\{f \in L^{2}(\Omega) \mid \int_{\Omega} f(x) \mathrm{d} x=0\right\}$ and $H^{1}(\Omega) / \mathbb{R}=H^{1}(\Omega) \cap L^{2}(\Omega) / \mathbb{R}$.

First we consider semigroup generation in $X$ for the solutions of (3.2) with no control or observation. When $\beta=0$, this semigroup generation is established in [2], see also Avalos and Lasiecka [4] for semigroup generation for the system with damping only on the active boundary. The proofs in $[2,4]$ can be very easily modified to show semigroup generation for the system described in (3.2).

In Avalos and Lasiecka [6] it is shown that for $\beta=0$, the control operator $\tilde{B}=$ $\left[\begin{array}{llll}0 & 0 & 0 & B\end{array}\right]^{T}$ (which corresponds to the control input $u$ ) is admissible for the system semigroup (see $[34,35]$ for some background on admissible control operators). The proof of the admissibility of $\tilde{B}$ can be easily modified for the case when $\beta>0$. The observation $y(t)$ in (3.2) is obtained via a bounded observation functional defined on $X$, so that this system is easily seen to be well-posed.

In Lasiecka [16], exponential stability is established for a structural acoustics system with a different damped beam equation on the active boundary, and in Avalos [3] exponential stability is established when the active boundary satisfies some geometric conditions and the damping is only on the active boundary. However, the systems in $[3,16]$ are sufficiently different from (3.2) that it is not obvious that the techniques used in these papers can be applied to (3.2). We briefly sketch here how the exponential stability of our system is proved. First we use the techniques in Avalos et al. [5] to establish that the map from the initial state to $\left[\begin{array}{ll}v & v_{t}\end{array}\right]^{T}$ is bounded from $X$ into $L^{2}\left([0, \infty),\left(H_{0}^{2}\left(\Gamma_{0}\right)\right)^{2}\right)$. We then use this, along with the exponential stability of the uncoupled wave equation with boundary damping (established in Lasiecka and Triggiani [17]), to show that the map from the initial state to $\left[z z_{t}\right]^{T}$ is bounded from $X$ into $L^{2}\left([0, \infty), H^{1}(\Omega) / \mathbb{R} \times L^{2}(\Omega) / \mathbb{R}\right)$. Hence, by a well known result of Datko [10], the semigroup of our system is exponentially stable.

The exponential stability of the system implies that $\mathbf{P}_{2} \in H^{\infty}\left(\mathbb{C}_{\alpha}\right)$ for some $\alpha<0$. A similar proof shows that $\mathbf{P}_{1}^{0}$, the transfer function from $d$ to $y$, is also in $H^{\infty}\left(\mathbb{C}_{\alpha}\right)$ (this is quite a bit easier, in fact, since $B_{1}$ is bounded while $B$ is unbounded). Hence $\mathbf{P} \in H^{\infty}\left(\mathbb{C}_{\alpha}\right)$ for some $\alpha<0$ and $\Sigma_{p}$ is a well-posed, exponentially stable system, so we can apply Theorem 1.1 to this system. 
We should say a bit about the condition that $\sigma\left(\mathbf{P}_{2}\left(i \omega_{j}\right) K_{j}\right) \in \mathbb{C}_{0}$. Clearly it will be possible to choose the gains $\left\{K_{j}\right\}_{j \in \mathcal{J}}$ so that this is satisfied if and only if $\mathbf{P}_{2}\left(i \omega_{j}\right) \neq 0$ at all of the frequencies $\omega_{j}$ which we wish to track. If the initial state is zero then (3.2) implies that

$$
\hat{y}(s)=s \int_{\Omega_{0}} \hat{z}(x, s) \mathrm{d} x .
$$

Since, by exponential stability, $\hat{z}(x, s)$ is bounded for $x \in \Omega_{0}$ and for $s$ near 0 , we see that $\mathbf{P}_{2}(0)=0$, so we cannot track constants. Since $\mathbf{P}_{2}$ is analytic and not identically equal to zero in $\mathbb{C}_{\alpha}$ for $\alpha<0$, we immediately conclude that the zeros of $\mathbf{P}_{2}$ on the imaginary axis have no finite accumulation point. Unfortunately, it is very difficult to compute $\mathbf{P}_{2}\left(i \omega_{j}\right)$ directly from (3.2); for this example the zeros of $\mathbf{P}_{2}$ would have to be estimated numerically.

Remark 3.1. We could of course take our observation to be multiple measurements of $z_{t}$ near $\left\{x_{i}\right\}_{i=1}^{p} \subset \Omega$. To guarantee well-posedness, we would take $y_{i}$ to be the integral of $z_{t}(t, x)$ over a small neighborhood of $x_{i}$. The objective would be to reduce the noise level near the $p$ points $x_{i}$. To satisfy (1.3), we would then need $p$ independent controls, hence $p$ patches on the active boundary.

\section{Disturbance rejection in a coupled beam}

In this section we consider two coupled Euler-Bernoulli beams, not necessarily of equal length, with the disturbance acting through a term distributed along the beams. Our goal is to design a controller, implemented as a dissipative joint, which stabilizes the system and rejects this disturbance. We assume that $\xi \in(0,1)$, the first beam has spatial extent $[0, \xi]$, the second beam has spatial extent $[\xi, 1]$, and the beams are hinged at 0 and 1. We assume that both are uniform Euler-Bernoulli beams with the same mass density per unit length $m$ and the same flexural rigidity $E I$. We normalize so that $E I / m=1$. Let $w(\zeta, t)$ be the displacement of the coupled beams at position $\zeta \in[0,1]$ and time $t>0$. The notation $\dot{w}(\zeta, t)$ denotes the derivative of $w(\zeta, t)$ with respect to time, and $D$ denotes the spatial differentiation operator. Then $w$ satisfies the following equations:

$$
\begin{array}{r}
\ddot{w}(\zeta, t)+D^{4} w(\zeta, t)=f(\zeta) d(t), \quad \zeta \in(0, \xi) \cup(\xi, 1) \\
w(0, t)=D^{2} w(0, t)=w(1, t)=D^{2} w(1, t)=0,
\end{array}
$$

where the disturbance $d(t)$ is of the form (3.1) and the spatial distribution of the disturbance is given by the function $f(\zeta)$. We assume for simplicity that $f \in L^{2}[0,1]$, but the development will work for some distributions $f$ as well.

Our goal is to design a dissipative joint at $\xi$ to stabilize this system and reject the disturbance. A natural first try would be to identify an input, an output and a 
feedback to realize a rigid support joint; see [9] for a discussion of joint and boundary conditions for coupled beams. This joint can be decribed mathematically by

$$
\begin{gathered}
w\left(\xi^{-}, t\right)=w\left(\xi^{+}, t\right), \quad D w\left(\xi^{-}, t\right)=D w\left(\xi^{+}, t\right), \quad D^{2} w\left(\xi^{-}, t\right)=D^{2} w\left(\xi^{+}, t\right), \\
D^{3} w\left(\xi^{+}, t\right)-D^{3} w\left(\xi^{-}, t\right)=-k \dot{w}(\xi, t), \quad k>0 .
\end{gathered}
$$

The appropriate well-posed input-output setting to realize (4.1), with this joint is given in [29]. Unfortunately, the exponential stability of this system is very nonrobust with respect to the joint position - see Theorem 1.1 in [29]. Similar comments apply to (4.1) with an angle guide joint:

$$
\begin{gathered}
w\left(\xi^{-}, t\right)=w\left(\xi^{+}, t\right), \quad D w\left(\xi^{-}, t\right)=D w\left(\xi^{+}, t\right), \quad D^{3} w\left(\xi^{-}, t\right)=D^{3} w\left(\xi^{+}, t\right), \\
D^{2} w\left(\xi^{-}, t\right)-D^{2} w\left(\xi^{+}, t\right)=-k D \dot{w}(\xi, t), \quad k>0 .
\end{gathered}
$$

In [1] it is shown that if both types of dissipative mechanisms are incorporated into the joint, then the system is exponentially stable independent of the joint position $\xi$. In particular, they consider the joint condition

$$
\begin{aligned}
& w\left(\xi^{-}, t\right)=w\left(\xi^{+}, t\right), \\
& D w\left(\xi^{-}, t\right)=D w\left(\xi^{+}, t\right) \\
& D^{3} w\left(\xi^{+}, t\right)-D^{3} w\left(\xi^{-}, t\right)=-\dot{w}(\xi, t), \quad D^{2} w\left(\xi^{-}, t\right)-D^{2} w\left(\xi^{+}, t\right)=-D \dot{w}(\xi, t),
\end{aligned}
$$

and show that for any $\xi \in(0,1),(4.1)-(4.2)$ is exponentially stable in the sense that $\left[w(\cdot, t), w_{t}(\cdot, t)\right]^{T}$ decays exponentially in the natural state space

$$
X=\left\{\left[x_{1}, x_{2}\right]^{T} \in H^{2}[0,1] \times L^{2}[0,1] \mid x_{1}(0)=x_{1}(1)=0\right\},
$$

which has inner product

$$
\left\langle\left[x_{1}, x_{2}\right]^{T},\left[y_{1}, y_{2}\right]^{T}\right\rangle=\int_{0}^{1}\left\{D^{2} x_{1}(\tau) D^{2} y_{1}(\tau)+x_{2}(\tau) y_{2}(\tau)\right\} d \tau
$$

We need to identify an input, output and feedback to realize (4.1)-(4.2) in such a way that we can apply Theorem 1.2 and the results in [1]. Upon first glace, the appropriate choices seem to be

$$
\begin{gathered}
D^{3} w\left(\xi^{+}, t\right)-D^{3} w\left(\xi^{-}, t\right)=u_{1}(t), \quad D^{2} w\left(\xi^{-}, t\right)-D^{2} w\left(\xi^{+}, t\right)=u_{2}(t), \\
z_{1}(t)=\dot{w}(\xi, t), \quad z_{2}(t)=D \dot{w}(\xi, t) .
\end{gathered}
$$

Unfortunately, in [29] it is shown that the transfer function from $u_{2}$ to $z_{2}$ is not well-posed. Therefore we use

$$
\begin{array}{r}
D^{3} w\left(\xi^{+}, t\right)-D^{3} w\left(\xi^{-}, t\right)=u_{1}(t), \quad D \dot{w}(\xi, t)=u_{2}(t), \\
z_{1}(t)=\dot{w}(\xi, t), \quad z_{2}(t)=D^{2} w\left(\xi^{-}, t\right)-D^{2} w\left(\xi^{+}, t\right) .
\end{array}
$$


We now show that (4.1) with (4.3) can be written as a well-posed system with state space $X$. Put

$$
A:=\left[\begin{array}{cc}
0 & I \\
-D^{4} & 0
\end{array}\right]
$$

with domain

$$
\begin{gathered}
\mathcal{D}(A)=\left\{\left[x_{1}, x_{2}\right]^{T} \in X \mid x_{1} \in H^{4}[0, \xi) \cup H^{4}(\xi, 1], x_{2} \in H^{2}[0,1],\right. \\
D^{2} x_{1}(0)=D^{2} x_{1}(1)=0, x_{2}(0)=x_{2}(1)=0, \\
\left.D x_{2}(\xi)=0, D^{3} x_{1}\left(\xi^{+}\right)=D^{3} x_{1}\left(\xi^{-}\right)\right\} .
\end{gathered}
$$

It is shown in [29, Section 3] that $A$ is a skew-adjoint operator which has a Riesz basis of eigenvectors on $X$, hence it generates a semigroup $S(t)$ on $X$. We need to identify the control operators associated with $u_{1}$ and $u_{2}$. To this end let $A_{1}$ be the extension of $A$ given by the same matrix of operators, with the following domain, which no longer requires $D x_{2}(\xi)=0$ and $D^{3} x_{1}\left(\xi^{+}\right)=D^{3} x_{1}\left(\xi^{-}\right)$, since these are boundary conditions in which the controls are incorporated:

$$
\begin{aligned}
\mathcal{D}\left(A_{1}\right)= & \left\{\left[x_{1}, x_{2}\right]^{T} \in X \mid x_{1} \in H^{4}[0, \xi) \cup H^{4}(\xi, 1], \quad x_{2} \in H^{2}[0,1],\right. \\
& \left.D^{2} x_{1}(0)=D^{2} x_{1}(1)=0, \quad x_{2}(0)=x_{2}(1)=0\right\}
\end{aligned}
$$

Using integration by parts, we obtain for $x=\left[x_{1}, x_{2}\right]^{T} \in \mathcal{D}\left(A_{1}\right)$ and $v=\left[v_{1}, v_{2}\right]^{T} \in$ $\mathcal{D}\left(A^{*}\right)=\mathcal{D}(A)$,

$$
\begin{aligned}
\left\langle A_{1} x, v\right\rangle & =\langle x,-A v\rangle+\left[D^{3} x_{1}\left(\xi^{+}\right)-D^{3} x_{1}\left(\xi^{-}\right)\right] v_{2}(\xi) \\
& +D x_{2}(\xi)\left[D^{2} v_{1}\left(\xi^{-}\right)-D^{2} v_{1}\left(\xi^{+}\right)\right]
\end{aligned}
$$

We extend $A$ to an operator from $X$ to $X_{-1}$ by

$$
\langle A x, v\rangle=\left\langle x, A^{*} v\right\rangle \text { for all } v \in \mathcal{D}\left(A^{*}\right)=\mathcal{D}(A) .
$$

Hence, (4.4) implies that for $x \in \mathcal{D}\left(A_{1}\right)$, the following equation holds in $X_{-1}$ :

$$
A_{1} x=A x+\left\{D^{3} x_{1}\left(\xi^{+}\right)-D^{3} x_{1}\left(\xi^{-}\right)\right\} B_{1}+D x_{2}(\xi) B_{2},
$$

where

$$
B_{1}=\left[\begin{array}{c}
0 \\
\delta(\cdot-\xi)
\end{array}\right], \quad B_{2}=\left[\begin{array}{c}
\delta^{\prime \prime}\left(\cdot-\xi^{-}\right)-\delta^{\prime \prime}\left(\dot{-} \xi^{+}\right) \\
0
\end{array}\right] .
$$

Let $B=\left[B_{1}, B_{2}\right], B_{d}=[0, f(\cdot)]^{T}$ and $u=\left[u_{1}, u_{2}\right]^{T}$. It is shown in [29] that $B_{2}$ is an admissible control operator for $A$, and it is easy to modify the arguments in [29] to show that $B_{1}$ is also admissible. The arguments in [29] can also be modified to show that (4.1), (4.3) determine a well-posed and regular system with feedthrough operator 0 . Therefore, the state space equations for (4.1) with (4.3) are

$$
\dot{x}(t)=A x(t)+B u(t)+B_{d} d(t), \quad z(t)=B_{\Lambda}^{*} x(t),
$$


where $B_{\Lambda}^{*}$ is the $\Lambda$-extension of $B^{*}$, as defined in [35].

Since $A$ is skew-adjoint, it is easy to see that the transfer function $\mathbf{Q}$ from $u$ to $z$ is positive and that $\operatorname{Re}(I+\mathbf{Q}(s)) \geq I$ for $s \in \mathbb{C}_{0}$, so $K=-I$ is an admissible feedback operator for (4.4) (see [35] for this concept). Therefore it follows that the feedback $u(t)=-z(t)$ in (4.1), (4.3) yields the well-posed exponentially stable system (4.1) with (4.2). Consider the controlled joint conditions and observation

$$
\begin{gathered}
w\left(\xi^{-}, t\right)=w\left(\xi^{+}, t\right), \quad D w\left(\xi^{-}, t\right)=D w\left(\xi^{+}, t\right), \\
D^{3} w\left(\xi^{+}, t\right)-D^{3} w\left(\xi^{-}, t\right)+\dot{w}(\xi, t)=u_{1}(t), \\
D^{2} w\left(\xi^{-}, t\right)-D^{2} w\left(\xi^{+}, t\right)+D \dot{w}(\xi, t)=u_{2}(t), \\
z_{1}(t)=\dot{w}(\xi, t), \quad z_{2}=D^{2} w\left(\xi^{-}, t\right)-D^{2} w\left(\xi^{+}, t\right) .
\end{gathered}
$$

The transfer function for the system described by (4.1), (4.5) and (4.6) is $\mathbf{P}_{2}(s)=$ $\mathbf{Q}(s)(I+\mathbf{Q}(s))^{-1}$, which is easily seen from this formula to be positive. We will apply Theorem 1.2 to this system.

We note that since $B_{d}$ is a bounded operator, the transfer function from $d$ to $z$ is well-posed. Therefore, to reject the disturbance while maintaining exponential stability, we can apply Theorem 1.2 to (4.1), (4.5), (4.6) as long as $\left\{\omega_{j}\right\}_{j \in \mathcal{J}} \subset \mathbb{R}$ is such that $\operatorname{Re} \mathbf{P}_{2}\left(i \omega_{j}\right)$ is invertible for all $j \in \mathcal{J}$. Let us examine this condition more closely. Since it is easy to verify that $\mathbf{Q}^{*}\left(i \omega_{j}\right)=-\mathbf{Q}\left(i \omega_{j}\right)$, we see that $\operatorname{Re} \mathbf{P}_{2}\left(i \omega_{j}\right)=-\left(\mathbf{Q}\left(i \omega_{j}\right)\right)^{2}\left(I-\left(\mathbf{Q}\left(i \omega_{j}\right)\right)^{2}\right)^{-1}$. Since $\operatorname{Re}\left(\mathbf{Q}\left(i \omega_{j}\right)\right)^{2} \leq 0$, we see that $\left(I-\left(\mathbf{Q}\left(i \omega_{j}\right)\right)^{2}\right)^{-1}$ is invertible for all $j \in \mathcal{J}$, so $\operatorname{Re} \mathbf{P}_{2}\left(i \omega_{j}\right)$ is invertible if and only if $\mathbf{Q}\left(i \omega_{j}\right)$ is invertible.

As an example, we consider the case when $\xi=1 / 2$. Write $s=i \eta^{2}$. In this case the transfer function is

$$
\mathbf{Q}(s)=\left[\begin{array}{cc}
\mathbf{Q}_{1,1}(s) & 0 \\
0 & \mathbf{Q}_{2,2}(s)
\end{array}\right]
$$

where

$$
\mathbf{Q}_{1,1}(s)=\frac{4 i}{\eta}\left\{\tan \frac{\eta}{2}-\tanh \frac{\eta}{2}\right\}, \quad \mathbf{Q}_{2,2}(s)=\frac{4}{i \eta} \frac{\sinh \frac{\eta}{2} \sin \frac{\eta}{2}}{\sin \frac{\eta}{2} \cosh \frac{\eta}{2}-\sinh \frac{\eta}{2} \cos \frac{\eta}{2}} .
$$

Thus, $\mathbf{Q}$ is nonsingular everywhere except at points where either $\mathbf{Q}_{1,1}(s)=0$ or $\mathbf{Q}_{2,2}(s)=0$. We have that $\mathbf{Q}_{1,1}(s)=0$ when $s= \pm i \eta_{1, k}^{2}$, where $\left\{\eta_{1, k}\right\}_{k=0}^{\infty}$ is asymptotic to $\{\pi / 2+2 \pi k\}_{k=0}^{\infty}$, and includes 0 . Furthermore, we can see that $\mathbf{Q}_{2,2}(s)=0$ when $s= \pm i \eta_{2, k}^{2}$, where $\left\{\eta_{1, k}\right\}_{k=0}^{\infty}=2 \pi+2 \pi k$. Thus, we can track signals containing components at all frequencies $\omega$ except $\left\{ \pm \eta_{1, k}^{2}, \eta_{2, k}^{2}\right\}_{k=0}^{\infty}$.

\section{References}

[1] K. Ammari, Z. Liu and M. Tucsnak, Decay rates for a beam with pointwise force and moment feedback, Mathematics of Control, Signals, and Systems, to appear. 
[2] G. Avalos, Well-posedness for a coupled hyperbolic/parabolic system seen in structural acoustics, IMA Preprint Series 1346, 1995.

[3] G. Avalos, The exponential stability of a coupled hyperbolic/parabolic system arising in structural acoustics, Abstract and Applied Analysis 1 (1996), pp. 203217.

[4] G. Avalos and I. Lasiecka, The strong stability of a semigroup arising from a coupled hyperbolic/parabolic system, Semigroup Forum 57 (1998), pp. 278-292.

[5] G. Avalos, I. Lasiecka, and R. Rebarber, Uniform decay properties of a model in structural acoustics, J. Math. Pures Appl. 79 (2000), pp. 1057-1072.

[6] G. Avalos and I. Lasiecka, Differential riccati equation for the active control of a problem in structural acoustics, J. Optim. Theory Applic. bf 91 (1996), pp. 695-728.

[7] H.T. Banks, W. Fang, R.J. Silcox and R.C. Smith, Approximation methods for control of structural acoustics models with piezoceramic actuators, Journal of Intelligent Material Systems and Structures 4 (1993), pp. 98-116.

[8] H.T. Banks and R.C. Smith, Feedback control of noise in a 2-D nonlinear structural acoustics model, Discrete and Continuous Dynamical Systems 1 (1995), pp. 119-149.

[9] G. Chen, M.C. Delfour, A.M. Krall, and G. Payre, Modeling, stabilization and control of serially connected beams, SIAM J. Control and Opt. 25 (1987), pp. 526546 .

[10] R. Datko, Extending a theorem of A. M. Liapunov to Hilbert Space, J. Math. Anal. Appl. 32 (1970), pp. 610-616.

[11] E.J. Davison, The robust control of a servomechanism problem for linear time invariant multivariable systems, IEEE Trans. Autom. Contr. 21 (1976), pp. 25-34.

[12] E.J. Davison, Multivariable tuning regulators: the feedforward and robust control of a general servomechanism problem, IEEE Trans. Autom. Contr. 21 (1976), pp. 35-47.

[13] B.A. Francis and W.M. Wonham, The internal model principle for linear multivariable regulators, Appl. Math. Optim. 2 (1975), pp. 170-194.

[14] T. Hämäläinen and S. Pohjolainen, Robust control and tuning problem for distributed parameter systems, Int. Jour. of Robust and Nonlinear Control 6 (1996), pp. 479-500.

[15] T. Hämäläinen and S. Pohjolainen, A finite dimensional robust controller for systems in the CD-algebra, IEEE Trans. Autom. Contr. 45 (2000), pp. 421-431. 
[16] I. Lasiecka, Boundary stabilization of a 3-dimensional structural acoustic model J. Math. Pure Appli. 78 (1999), pp. 203-232.

[17] I. Lasiecka and R. Triggiani, Uniform stabilization of the wave equation with Dirichlet or Neumann feedback control without geometrical condtions, Appl. Math. Optim. 25 (1992), pp. 189-224.

[18] H. Logemann, Stabilization and regulation of infinite-dimensional systems using coprime factorizations, pp. 102-139 in Analysis and Optimization of Systems: State and Frequency Domain Approaches for Infinite-Dimensional Systems, Editors: R.F. Curtain, A. Bensoussan and J.L. Lions, LNCIS vol. 185, SpringerVerlag, Berlin, 1993.

[19] H. Logemann, J. Bontsema, and D.H. Owens, Low-gain control of distributed parameter systems with unbounded control and observation, Control Theory Advanced Tech. 4 (1988), pp. 429-446.

[20] H. Logemann and R.F. Curtain, Absolute stability results for infinite-dimensional well-posed systems with applications to low-gain control, ESAIM: Control, Optimisation and Calculus of Variations 5 (2000), http://www.edpsciences.com/articles/cocv/abs/2000/01/cocvVol5-16/cocvVol5-16.html.

[21] H. Logemann and A.D. Mawby, Low-gain integral control of infinite dimensional regular linear systems subject to input hysteresis, in "Advances in Mathematical Systems Theory" (edited by F. Colonius et al.), Birkhäuser. Boston, 2001., pp. 255-293.

[22] H. Logemann and D.H. Owens, Low-gain control of unknown infinite-dimensional systems: A frequency-domain approach, Dynamics and Stability of Systems 4 (1989), pp. 13-29.

[23] H. Logemann, E.P. Ryan and S. Townley, Integral control of infinite-dimensional linear systems subject to input saturation, SIAM J. Control Optim. 36 (1998), pp. 1940-1961.

[24] H. Logemann and S. Townley, Low-gain control of uncertain regular linear systems, SIAM J. Control and Optim. 35 (1997) pp. 78-116.

[25] H. Logemann and S. Townley, Discrete-time low-gain control of uncertain infinite-dimensional system, IEEE Trans. Auto. Control, 42 (1997), pp. 22-37.

[26] S. Pohjolainen, Robust multivariable PI-controllers for infinite dimensional systems, IEEE Trans. Autom. Control 27 (1982), pp. 17-30.

[27] S. Pohjolainen, Robust controllers for systems with exponentially stable strongly continuous semigroups, J. Math. Anal. Appl. 111 (1985), pp. 622-636.

[28] S. Pohjolainen and I. Lätti, Robust controller for boundary control systems, Int. J. Control 38 (1983), pp. 1189-1197. 
[29] R. Rebarber, Exponential stability of coupled beams with dissipative joints: a frequency domain approach, SIAM J. Control and Opt. 33 (1995), pp. 1-28.

[30] D. Salamon, Infinite dimensional systems with unbounded control and observation: A functional analytic approach, Transactions of the Amer. Math. Society 300 (1987), pp. 383-431.

[31] D. Salamon, Realization theory in Hilbert space, Math. Systems Theory 21 (1989), pp. 147-164.

[32] O.J. Staffans, Quadratic optimal control of stable well-posed linear systems, Trans. Amer. Math. Soc. 349 (1997), pp. 3679-3715.

[33] O.J. Staffans, Admissible factorizations of Hankel operators induce well-posed linear systems, Systems and Control Letters, 37:301-307, 1999.

[34] G. Weiss, Transfer functions of regular linear systems, Part I: Characterizations of regularity, Trans. Amer. Math. Society 342 (1994), pp. 827-854.

[35] G. Weiss, Regular linear systems with feedback, Mathematics of Control, Signals, and Systems, 7 (1994), pp. 23-57.

[36] G. Weiss and R.F. Curtain, Dynamic stabilization of regular linear systems, IEEE Trans. Aut. Control 42 (1997), pp.4-21.

[37] G. Weiss and R. Rebarber, Optimizability and estimatability for infinitedimensional linear systems, SIAM J. Control and Opt. 39 (2001), pp. 1204-1232. 